\title{
The Link Between the Formation Rates of Clusters and Stars in Galaxies
}

\author{
Rupali Chandar, ${ }^{1}$ S. Michael Fall, ${ }^{2}$ and Bradley C. Whitmore ${ }^{2}$
}

\begin{abstract}
The goal of this paper is to test whether the formation rate of star clusters is proportional to the star formation rate (SFR) in galaxies. As a first step, we present the mass functions of compact clusters younger than $10 \mathrm{Myr}$ in seven star-forming galaxies of diverse masses, sizes, and morphologies: the Large and Small Magellanic Clouds, NGC 4214, NGC 4449, M83, M51, and the Antennae. These cluster mass functions (CMFs) are well represented by power laws, $d N / d M \propto M^{\beta}$, with similar exponents $\beta=-1.92 \pm 0.27$, but with amplitudes that differ by factors up to $\sim 10^{3}$, corresponding to vast differences in the sizes of the cluster populations in these galaxies. We then normalize these CMFs by the SFRs in the galaxies, derived from dust-corrected $\mathrm{H} \alpha$ luminosities, and find that the spread in the amplitudes collapses, with a remaining rms deviation of only $\sigma(\log A)=0.2$. This is close to the expected dispersion from random uncertainties in the CMFs and SFRs. Thus, the data presented here are consistent with exact proportionality between the formation rates of stars and clusters. However, the data also permit weak deviations from proportionality, at the factor of two level, within the statistical uncertainties. We find the same spread in amplitudes when we normalize the mass functions of much older clusters, with ages in the range 100 to $400 \mathrm{Myr}$, by the current SFR. This is another indication of the general similarity among the cluster populations of different galaxies.
\end{abstract}

Subject headings: galaxies: individual (LMC, SMC, NGC 4214, NGC 4449, M83, M51, Antennae) — galaxies: star clusters — stars: formation

\section{Introduction}

Stars form together in clusters and associations, which in turn form in the densest parts - the clumps - of molecular clouds (Lada \& Lada 2003; McKee \& Ostriker 2007). There

\footnotetext{
${ }^{1}$ Department of Physics \& Astronomy, The University of Toledo, Toledo, OH 43606

${ }^{2}$ Space Telescope Science Institute, Baltimore, MD, USA
} 
is some debate about the exact definition of a cluster, e.g., whether this term refers to all dense stellar aggregates or only those that are gravitationally bound or free of gas. Nevertheless, there appears to be unanimous agreement that stars mostly form in aggregates of some sort and rarely in isolation. This general picture implies that the formation rates of stars and clusters should be proportional to each other. The purpose of this paper is to present a direct test of this proportionality.

Our approach is to compare the mass functions, $\psi(M)=d N / d M$, of recently formed star clusters (with ages $\tau<10 \mathrm{Myr}$ ) in different galaxies before and after normalizing by the star formation rates in the galaxies. This is valid because the mass functions of young clusters in different galaxies have similar power-law shapes, $\psi(M) \propto M^{\beta}$ with $\beta \approx-2$, over large intervals of mass, roughly $10^{3} M_{\odot} \lesssim M \lesssim 10^{6} M_{\odot}$ (Fall \& Chandar 2012 and references therein). The mass functions are unknown at lower masses, as a result of selection limits in the available samples, and are uncertain at higher masses as a result of small number statistics. This is the reason that we compare the mass functions, rather than the total numbers or total masses of young clusters (the integrals of $\psi(M)$ and $M \psi(M)$ respectively over all $M$ ), with the star formation rates.

In this work, we use the term "cluster" for any concentrated aggregate of stars with a density much higher than that of the surrounding stellar field, whether or not it is gravitationally bound. It is not possible to tell from images alone which clusters are gravitationally bound (have negative energy) and which are unbound (have positive energy), and N-body simulations show that unbound clusters retain the appearance of bound clusters for many $(\sim 10)$ crossing times (Baumgardt \& Kroupa 2007). While spectra might help in principle, they are seldom available and are often contaminated by non-virial motions (stellar winds, binary stars, etc).

In the next section (Section 2), we present the catalogs and mass functions in seven galaxies with well-studied populations of clusters. In the following section (Section 3), we estimate the star formation rates, determine the CMF/SFR relations, present a method for comparing this relation between galaxies, and compare the residuals with various galaxy properties. In the last section (Section 4), we summarize some implications of our results for the formation and disruption of the clusters.

\section{Cluster Catalogs and Mass Functions}

Our study focuses on seven nearby galaxies: LMC, SMC, NGC 4214, NGC 4449, M83, M51, and the Antennae. In this section, we collect and describe the star cluster catalogs, 
and summarize the method used to estimate the masses and ages of the clusters. We then present the mass functions of the clusters in each galaxy.

\subsection{Star Cluster Samples}

We include nearby galaxies with published star cluster catalogs that cover at least $50 \%$ of the optically luminous portions of the galaxy and have photometry in at least four optical passbands including the $U$ band (which is critical for disentangling the effects of age and extinction in the colors of young clusters). Our sample includes galaxies of different types: irregular, spiral, and merger, which span a large range in distance, luminosity, color, inclination, and star formation rate. This sample, while small, is reasonably representative of nearby star-forming galaxies in general. The observations, data, and cluster selection criteria for each galaxy are summarized below.

Large and Small Magellanic Clouds: We use the catalogs published by Hunter et al. (2003), where clusters were selected by visual examination of candidates (from previously published catalogs). The clusters were distinguished from the surrounding distribution of field stars, resolved with respect to a single star, but do not include low density H II regions. The observations cover approximately $70 \%$ and $90 \%$ of the recent star formation in each galaxy, as traced by $\mathrm{H} \alpha$ emission. This resulted in a total of 854 (239) clusters in the LMC (SMC) with published UBVR photometry.

NGC 4214: This dwarf irregular galaxy, at a distance of 3.1 Mpc (Dopita et al. 2010), was observed in a single pointing with the WFC3 on-board the Hubble Space Telescope (HST) as part of program 11360 (PI: O'Connell). Clusters were automatically selected to be compact sources broader than the PSF followed by elimination of close pairs of stars, which resulted in a total of 334 candidate clusters. Aperture photometry was performed on $U B V I \mathrm{H} \alpha$ images in a manner similar to that described in Chandar et al. (2010) for sources in the nearby spiral galaxy M83.

NGC 4449: Rangelov et al. (2011) published a catalog of 129 clusters in this dwarf starburst galaxy (located at a distance of $3.82 \pm 0.27 \mathrm{Mpc}$; Annibali et al. 2008) based on HST observations. They selected cluster candidates automatically using size criteria, followed by a visual inspection to eliminate contaminants such as background galaxies and chance superpositions. Two fields within NGC 4449 were observed with the ACS/WFC in the $B V I \mathrm{H} \alpha$ bands, and two in the $U$ filter with the WFPC2 camera, giving near-complete coverage of the optically luminous portions of the galaxy. 
M83: Two fields within M83, a grand-design spiral galaxy located at a distance of 4.5 Mpc (Thim et al. 2003), were observed as part of program GO-11360. Several cluster catalogs were produced for each field, according to the selection methodology described in Chandar et al. (2014). Here, we adopt the manual cluster catalogs from that work. We also use data from five additional fields in M83 that were observed with the HST/WFC3 as part of program GO-12513, bringing the coverage of the optically luminous portion of M83 to $\approx 60 \%$. New cluster catalogs have been produced for these fields, and will be presented in Whitmore et al. (in prep). Our final catalog contains 3186 star clusters.

M51: Most of the optically luminous portions of this grand-design spiral galaxy, located at a distance of $8.2 \mathrm{Mpc}$, were observed in six fields taken with the $H S T /$ ACS camera in $B V I \mathrm{H} \alpha$, and in 8 overlapping fields with the WFPC2 camera in the $U$ band. Just as for NGC 4449, star clusters were selected using automated criteria, followed by visual examination. This resulted in a catalog of 3812 clusters (Chandar et al., in prep).

Antennae: The merging Antennae galaxies, located at a distance of $21 \mathrm{Mpc}$ (Schweizer et al. 2008), were observed with the ACS/WFC on the HST as part of program GO-10188, in the $B V I \mathrm{H} \alpha$ filters (Whitmore et al. 2010). Additional observations were taken in the $U$ band with the WFC3 camera as part of program GO-11577. Clusters were selected to be objects brighter than $M_{V}=-9$, a restriction that eliminates practically all individual stars.

The largest source of uncertainty in these catalogs is in the selection of clusters with ages $\tau \lesssim 10$ Myr. For the Magellanic Clouds, it is possible that clusters that are both very young and/or have low stellar density were excluded by the selection criteria. For the more distant galaxies, very young clusters might be excluded because they are very compact and their profiles are indistinguishable from individual stars, but on the other hand, chance superpositions of unassociated young stars might sometimes be selected as young clusters. The selection of compact clusters older than $10 \mathrm{Myr}$ is more robust (as discussed in Chandar et al. 2014), because by this age clusters have typically moved away from their crowded birth sites.

\subsection{Mass and Age determinations}

For each cluster in each galaxy, the measured magnitudes, in the filters listed above, were compared with predictions from the Bruzual \& Charlot (2003, 2010) stellar population models, in order to estimate their age, extinction, and mass.

We first estimate the age $\tau$ and the extinction $A_{V}$ by performing a $\chi^{2}$ fit comparing observed and predicted magnitudes, assuming a Chabrier (2003) IMF, and a Galactic-type 
extinction law (Fitzpatrick 1999). The best-fit values of $\tau$ and $A_{V}$ are those that minimize:

$$
\chi^{2}\left(\tau, A_{V}\right)=\sum_{\lambda} W_{\lambda}\left(m_{\lambda}^{\mathrm{obs}}-m_{\lambda}^{\mathrm{mod}}\right)^{2},
$$

where $m_{\lambda}^{\text {obs }}$ and $m_{\lambda}^{\text {mod }}$ are the observed and model magnitudes respectively, and the sum runs over all filters listed above. The weight factors in the formula for $\chi^{2}$ are taken to be $W_{\lambda}=\left[\sigma_{\lambda}^{2}+(0.05)^{2}\right]^{-1}$, where $\sigma_{\lambda}$ is the photometric uncertainty.

The mass of each cluster is estimated from the extinction-corrected $V$-band magnitude, the distance listed in Table 1, and the mass-to-light ratio $\left(M / L_{V}\right)$ predicted by the models at the fitted ages $\tau$. The models assume that the stellar IMF for each cluster is fully sampled. While the colors of clusters with masses below $\approx 10^{4} M_{\odot}$ begin to spread around the mean values because the upper end of the stellar IMF is not fully populated, the resulting mass distributions, down to at least $\approx 3 \times 10^{3} M_{\odot}$, are very similar regardless of whether fully or stochastically sampled models are used (Fouesneau et al. 2012).

The largest uncertainties in the mass estimates comes from uncertainties in the age estimates, which are typically on the order of 0.3 in $\log \tau$, corresponding to a factor of $\approx 2$ in $\tau$. These translate to approximately similar uncertainties of 0.3 and $\approx 2$ in $\log M$ and $M$. There are also two sources of systematic uncertainty. Any error in a galactic distance translates directly into a fractional change in the masses of all clusters within that galaxy. The derived masses of the clusters, but not their ages, also depend on the IMF assumed in the stellar population models. For example, if we had assumed a Salpeter (1955) instead of Chabrier IMF, the estimated mass of each cluster would increase by a nearly constant $\approx 40 \%$. Similarly, we find that a mix of stellar IMFs between Chabrier and Salpeter would increase the estimated masses by a nearly constant fractional amount between 0 and $40 \%$ (depending on the exact mix of IMFs). Such fractional changes in $M$, corresponding to shifts in $\log M$, would not alter the shape of the cluster mass function (in log-log plots).

\subsection{Cluster Mass Functions}

In the left-hand panels of Figures 1 and 2, we show the mass functions determined for the clusters in all seven of our sample galaxies in two different intervals of age: recently formed $(\tau<10 \mathrm{Myr})$ clusters, which are the main focus of this paper, and also intermediate-age $(\tau=100-400 \mathrm{Myr})$ clusters as an interesting check. An equal number of clusters have been placed in each bin, from 3 to 7 , depending on the available sample size (resulting in variable bin widths). The low end of the mass function in each galaxy is set by the luminosity limit of each catalog, while the high end of these distributions is not constrained. The amplitudes 
of the cluster mass functions directly reflect differences in the sizes of the cluster populations among the galaxies. There is clearly a large range, approximately a factor of $10^{3}$, in these amplitudes.

The merging Antennae system has the highest number of stellar clusters in our sample (in both age intervals). It is followed by the spirals M51 and M83, then the luminous starforming irregular galaxies NGC 4449 and the LMC, with the low luminosity irregular galaxies NGC 4214 and the SMC having the fewest detected clusters. The high end of the cluster mass functions also generally correlate with the total number of clusters, in the sense that the galaxies with the most clusters tend to have the most massive clusters.

The observed mass functions are well represented by featureless power laws: $d N / d M \propto$ $M^{\beta}$. Thus, we perform linear fits to $\log (d N / d \log M)=(\beta+1) \log M+$ const. The bestfit exponents and their formal $1 \sigma$ errors are listed in Table 1 . Despite the very different types of galaxies included in our sample, different cluster selection criteria, filter sets, etc., the resulting exponents have a fairly narrow range, $\beta=-1.92 \pm 0.27$, indicating that the mass functions of young star clusters in nearby star-forming galaxies have an approximately "universal" shape.

Uncertainties in the CMF are dominated by the selection of the clusters. For clusters younger than $10 \mathrm{Myr}$, we estimate that the uncertainties in the amplitude of the CMF could be as large as $\approx 0.1$ based on comparisons of the cluster catalogs from different groups, which differ at the 30-40\% level in this age range (e.g., Bastian et al. 2012; Chandar et al. 2014). The uncertainty for 100-400 Myr clusters is smaller, roughly $\approx 0.04$ because cluster catalogs in this age range only differ by $\approx 10 \%$ between groups.

\section{Star Formation Rates and Normalized Mass Functions}

\subsection{Star Formation Rates}

We determine the star formation rate for each galaxy in our sample from its $\mathrm{H} \alpha$ line emission. Most of this emission ( $90 \%$ ) is contributed by stars younger than 10 Myr (Kennicutt \& Evans 2014). Therefore, $\mathrm{H} \alpha$ emission measures the SFR over the same age range as the clusters in our younger subsample. We use the most recent $\mathrm{H} \alpha$ flux measurements listed in NED and corrected for contamination by [N II] emission, and assume the distances given in column 3 of Table 1 when converting to $\mathrm{H} \alpha$ luminosities.

The $\mathrm{H} \alpha$ emission is attenuated by dust. To correct for this attenuation, we use the measured $25 \mu \mathrm{m}$ flux (available for all galaxies in our sample), and the recipe given in 
Table 2 in Kennicutt \& Evans (2014):

$$
L(H \alpha)_{\mathrm{corr}}=L(\mathrm{H} \alpha)_{\mathrm{obs}}+0.020 L(25 \mu m) .
$$

We then adopt their Equation (12) and Table 1 to determine the star formation rate:

$$
\log \operatorname{SFR}\left(M_{\odot} / \mathrm{yr}\right)=\log L(\mathrm{H} \alpha)_{\mathrm{corr}}-41.27 .
$$

This calibration is based on the STARBURST99 models (Leitherer et al. 1999) assuming solar metallicity, a Kroupa IMF (similar to the Chabrier IMF in the Bruzual \& Charlot models), and a constant rate of star formation. The resulting SFRs are compiled in column 5 of Table 1.

There are several inherent sources of uncertainty in $\mathrm{H} \alpha$-based star formation rate determinations, including flux measurements, corrections made for attenuation by dust, variations in the star formation rate, uncertainties in the calibration used to convert the measured fluxes to SFR as discussed in the review by Kennicutt \& Evans (2014), and the leakage of Lyman continuum photons from the parent galaxy. Estimates of these uncertainties are listed in Table 2. For our sample specifically, we find that the published $\mathrm{H} \alpha$ and $25 \mu \mathrm{m}$ flux measurements (compiled in NED) used to determine the SFRs, typically differ by $\approx 20 \%$, although one galaxy has published values that differ by $\approx 50 \%$. Different methods to correct for dust extinction introduce uncertainties of $\approx 20-30 \%$, based on a comparison between a linear combination of the $\mathrm{H} \alpha$ and $24 \mu \mathrm{m}$ luminosities with Balmer-decrement corrected $\mathrm{H} \alpha$ luminosities (Figure 3, Kennicutt \& Evans 2014), although the uncertainties can be higher for dusty galaxies like the Antennae. We estimate that the uncertainty in the fractional coverage of each galaxy is $\approx 10 \%$. Lyman continuum photons can escape from their parent galaxy, further increasing the uncertainty in the SFR determinations, although the escape fraction can vary strongly from one galaxy to another and is therefore difficult to quantify. Taken together, the random uncertainties related to the SFR determinations and coverage correspond to at least $\approx 0.2$ in the logarithm of the CMF/SFR relation, not including uncertainties related to the cluster mass functions. Systematic uncertainties may also be present, since stellar population modeling shows that the calibrations likely overestimate the SFR for lower metallicity populations, because the ionizing luminosity increases by $\sim 0.4 \pm 0.1$ dex for a tenfold decrease in the metallicity (e.g., Kennicutt \& Evans 2014; Smith, Norris, \& Crowther 2002; Raiter, Schaerer \& Fosbury 2010).

Our $\mathrm{H} \alpha$-based star formation rates agree reasonably well with previous determinations published in the literature (compiled in Column 6 of Table 1). Lee et al. (2009) determined H $\alpha$-based SFRs for NGC 4214, NGC 4449, M83, and M51, but used a different method to correct for dust and a different calibration to convert $\mathrm{H} \alpha$ luminosity to SFR (taken from Kennicutt 1998). James et al. (2008) estimated H $\alpha$-based SFRs for the Large and Small 
Magellanic Cloud using the prescription given by Kennicutt (1998), and Zhang et al. (2001) measured the $\mathrm{H} \alpha$ line intensity from $H S T$ /WFPC2 images and applied the prescription from Kennicutt (1998) to determine the SFR of the Antennae. In Column 7 of Table 1, we list previously published star formation rates determined for each galaxy using various non-H $\alpha$ based methods. Overall, we find that our SFR determinations agree with published values to somewhat better than a factor of two, which is consistent with the uncertainties found by Lee et al. (2009) by comparing far ultraviolet and H $\alpha$-based SFRs for a much larger sample of galaxies.

\subsection{The CMF/SFR Relation}

The right panel of Figure 1 presents the main result of this paper. The same cluster mass functions as in the left panel are shown, but these distributions have now been divided by the star formation rate (listed in Column 5 of Table 1) of the host galaxy within the area covered by each cluster catalog: CMF/SFR. The mass functions of star clusters in these very different galaxies all have very similar amplitudes when normalized by the star formation rate of their host galaxies.

In Figure 2, we again show the observed and SFR-normalized cluster mass functions, but now for clusters with ages between 100 and 400 Myr. Evidently, the mass functions of these intermediate-age clusters also lie very close to one another in the vertical direction after they have been normalized by the current star formation rate of the host galaxy. This is remarkable because the SFR-normalized mass function of intermediate-age clusters can be affected by variations in the past formation and disruption rates of the clusters. The similarity of these CMFs indicates that the SFRs were relatively steady for the past $400 \mathrm{Myr}$, and that the cluster disruption rates were similar among the galaxies in our samples.

In order to quantify the observed scatter, we fit the CMF/SFR for each galaxy by the function:

$$
d N / d M=A \times \operatorname{SFR} \times\left(M / 10^{4} M_{\odot}\right)^{-1.9}
$$

where we have fixed the power-law exponent to -1.9 (the mean of all $\beta$ values) for all galaxies. We use our $\mathrm{H} \alpha$-based SFRs from Table 1, adjust them for partial coverage (if needed), then normalize the distributions at $10^{4} M_{\odot}$, which is about the middle of the cluster log mass range observed across the galaxies. The coefficient $A$ measures the proportionality between the cluster and star formation rates. The dispersion $\sigma(\log A)$ in the best fit values of $\log A$, quantifies the scatter in the amplitudes and hence in the CMF/SFR relation among galaxies.

We demonstrate our technique in Figure 3. The SFR-normalized cluster mass functions 
are shown in two intervals of age: $\tau<10$ Myr (left panel) and $\tau=100-400 \mathrm{Myr}$ (right panel). The lines show the best fit from equation (4), and the intercept values $A$ are the values where each fit intersects the dotted line. For the seven galaxies studied here, we find that $\sigma(\log A)=0.21$ for clusters with ages $\tau<10$ Myr. The scatter for intermediateage $(100-400 \mathrm{Myr})$ clusters is similar, with $\sigma(\log A)=0.20$. This scatter is close to the dispersion expected from random uncertainties in the CMFs and the SFRs, as discussed in Sections 2.3 and 3.1 .

The data presented here are consistent with exact proportionality between the formation rates of stars and clusters. Nevertheless, within the uncertainties, the data also allow for weak deviations, at the approximately factor of two level. In Figure 4, we search for trends in the residuals of $A$ with host galaxy properties, including SFR, luminosity, color, inclination, maximum rotational velocity of the gas, and the exponent of the cluster mass function $\beta$. The nonparametric Spearman correlation coefficients, $r_{S}$ and the corresponding probabilities $\mathrm{p}\left(>r_{S}\right)$ associated with each plot are compiled in Table 3. Values for $\mathrm{p}\left(>r_{S}\right)$ should be less than 0.05 to have greater than $95 \%$ confidence that a correlation is real. For the young $(\tau<10 \mathrm{Myr})$ clusters, we do not observe any statistically significant correlations. For the intermediate-age (100-400 Myr old) clusters, there are weak positive trends between the residuals of $\log A$ and galactic SFR, luminosity, and $B-V$ color, but only the one with $B-V$ is statistically significant (at the $95 \%$ level). Possibly, these trends may reflect differences in the metallicities of the galaxies. In any case, a larger sample of galaxies is needed to determine whether or not such trends are real (e.g., the LEGUS project; Calzetti et al. 2015). We find no statistically significant correlation for clusters in either age range between the residuals of $\log A$ and the SFR per unit area or SFR density (not shown), which is often used to compare cluster populations among galaxies.

\subsection{Comparison with Previous Work}

The CMF/SFR statistic studied here is closely related to the cluster formation efficiency $\Gamma$, defined as the fraction of stellar mass formed in bound clusters. Estimates of $\Gamma$ in numerous studies have been used to draw important conclusions about the properties of star-forming molecular clouds in different galaxies and environments. Since, as discussed in the Introduction, it is not possible to distinguish bound from unbound clusters based on images alone, $\Gamma$ should be directly proportional to our CMF/SFR statistic.

$\Gamma$ has been estimated for a number of galaxies, using various methods, and large differences are claimed from one galaxy to another. All of these studies integrate over the CMF in order to determine the total mass in clusters, but use different assumptions, extrapolations, 
age and mass ranges, and methods to accomplish this (e.g., Goddard et al. 2010; Adamo et al. 2011; Silva-Villa \& Larsen 2011; Silva-Villa et al. 2013). The compiled results (e.g., Kruijssen 2012) show a strong increase in $\Gamma$, by a factor of $\sim 15$, for galaxies with increasing star formation rate densities, from $\approx 10^{-3}$ to $\approx$ a few $M_{\odot} \mathrm{yr}^{-1} \mathrm{kpc}^{-2}$ (corresponding to the $\sim 3$ dex range of SFRs from $\sim 0.05$ to $\sim 50 M_{\odot} \mathrm{yr}^{-1}$ ). The corresponding relation between $\Gamma$ and SFR (which has a similar slope when transformed from the original SFR density given in Kruijssen 2012) is shown as the dashed line in the top-left panel of Figure 4. This trend is much stronger than our CMF/SFR relation for young $(\tau<10 \mathrm{Myr})$ and intermediate-age (100-400 Myr) clusters.

This may be due to the fact that estimates of $\Gamma$ are sensitive to the specific assumptions and extrapolations that are made 1 and are thus prone to large errors. For example, Goddard et al. (2010) found a significantly higher value of $\Gamma$ for clusters in the Antennae when compared with those in the Magellanic Clouds, while we find similar CMF/SFR relations for these three galaxies. Our results are based on a larger cluster catalog for the Antennae, but the same ones as used in Goddard et al. (2010) for the LMC and SMC. The dotted line in the top-left panel of Figure 4 shows that the theoretical prediction for $\Gamma$ versus SFR from Kruijssen (2012) is much steeper than the observations, and therefore disagrees even more strongly with our results for the CMF/SFR statistic.

We have presented a robust new method for comparing the fraction of stars that form in clusters, one that only relies on comparing the amplitudes of the CMF normalized by the SFR in galaxies. The parameter $\Gamma$ has been extensively used in the literature, but requires additional assumptions and extrapolations that degrade its reliability. We also advocate that, as done here, the SFR rather than the more uncertain SFR density be used when comparing results among galaxies. There is no standard method for estimating the starforming area within galaxies, and we find that the uncertainties in area alone can be as large as a factor of a few (see also discussion in Adamo et al. 2011).

\section{Conclusions and Implications}

The main conclusion of this paper is that star and cluster formation rates are proportional to each other in a diverse but representative sample of nearby galaxies. There are only minor deviations from exact proportionality in our sample (with an rms scatter less

\footnotetext{
${ }^{1}$ In particular, in this approach the extrapolated number of clusters depends sensitively on the fit and uncertainties in the power-law index $\beta$ and also on the exact mass and age ranges that are adopted. Small uncertainties in any of these values can drastically change the results.
} 
than a factor of 2) in two different age ranges, and no trends are observed in the residuals of CMF/SFR with galaxy property for young $(\tau<10 \mathrm{Myr})$ clusters. For the intermediateage (100-400 Myr) clusters, weak trends with the star formation rate and luminosity of the host galaxy may be present, but it is also possible that systematic uncertainties in the SFR determinations are responsible. A similar study with a larger sample is needed to establish whether or not weak trends exist.

Our results are consistent with previous findings that the luminosity of the brightest cluster in a galaxy is approximately proportional both to the number of clusters in the galaxy (e.g., Whitmore 2003) and to its star formation rate (Larsen 2002; Bastian 2008). However, inferences based only on the brightest cluster in a galaxy require extra (though plausible) assumptions about all the other, fainter clusters: their mass function, mass-tolight ratios, interstellar extinctions, and so forth. The method presented here is much more robust because it is based on the mass functions determined from hundreds or thousands of clusters over a wide range of masses and specific, narrow ranges of age $(\tau<10 \mathrm{Myr}$ and 100-400 Myr).

Our results are also consistent with our previous findings that the mass and age distributions of clusters are similar in different galaxies. We approximate the joint distribution by a double power law: $g(M, \tau) \propto M^{\beta} \tau^{\gamma}$ with $\beta \approx-2$ and $-1.0 \lesssim \gamma \lesssim-0.5$ (Fall \& Chandar 2012, Chandar et al. 2014). We refer to this as a quasi-universal distribution because the observed exponents differ relatively little among galaxies (typically, $\Delta \beta \sim \Delta \gamma \sim 0.2$ ). 2 Our results for the $\mathrm{CMF} / \mathrm{SFR}$ relation imply that the populations of clusters on galaxy scales are nearly the same apart from an overall normalization factor. The results presented here indicate that this normalization factor is the star formation rate.

Our claim that populations of clusters are similar in different galaxies might seem to be contradicted by the fact that massive clusters are found in some galaxies but not in others. For example, the most massive clusters in the Antennae have $M_{\max } \sim 10^{7} M_{\odot}$ whereas those in the SMC have $M_{\max } \sim 10^{4} M_{\odot}$ (see Figure 1). However, this is largely, if not entirely, due to the different sizes of these samples or populations, roughly $10^{3}$ times larger in the Antennae than in the SMC. Indeed, it is easy to show that, for the quasi-universal model, the maximum cluster mass scales with the population size and hence with the star

2 While the mass and age distributions of star clusters are remarkably similar on galaxy scales, in small regions there can be large variations in the formation and disruption rates, and hence in the observed mass and age distributions of the clusters (see e.g., Bastian et al. 2012; Silva-Villa et al. 2014; Chandar et al. 2014 for an example of this effect in M83). As these regions are combined into larger ones, the variations will average out and the differences diminish. In particular, the exponent $\gamma$ of the age distribution may vary significantly with galactocentric distance for the physical reasons discussed by Fall \& Chandar (2012). 
formation rate as $M_{\max } \propto S F R^{-1 /(\beta+1)}$, where $\beta$ again is the exponent of the mass function; thus $M_{\max } \propto \mathrm{SFR}$ for $\beta=-2$.

The quasi-universal model implies that the clusters in different galaxies are formed and disrupted in similar (although not exactly the same) ways. This is a remarkable fact, probably the consequence of a great deal of similarity in the structure of the interstellar media among galaxies on the scale of molecular clumps and clouds, in particular in their mass functions, which play roles in both the formation and disruption of clusters (see Fall, Krumholz, \& Matzner 2010 and Fall \& Chandar 2012 for further discussion of these topics). Until recently, it has not been possible to observe the ISM of other galaxies with sufficient angular resolution to test this conjecture, but with the advent of ALMA, this may now be within reach.

R.C. acknowledges support from NSF through CAREER award 0847467. S.M.F. acknowledges the hospitality of the Aspen Center for Physics, which is supported in part by the National Science Foundation under grant PHYS-1066293. We thank the anonymous referee and Mark Krumholz for suggestions that improved our paper.

Facilities: HST.

\section{REFERENCES}

Adamo, A., Ostlin, G., \& Zackrisson, E. 2011, MNRAS, 417, 1904

Annibali, F., Aloisi, A., Mack, J., et al. 2008, AJ, 135, 1900

Bastian, N. 2008 MNRAS, 390, 759

Bastian, N., Adamo, A., Gieles, M., Silva-Villa, E., Lamers, H. J. G. L. M., Larsen, S. S., Smith, L. J., Konstantopoulos, I. S., \& Zackrisson, E. 2012, MNRAS, 419, 2606

Bolatto, A. D., Leroy, A. K., Jameson, K., et al. 2011, ApJ, 741, 12

Bruzual, G., \& Charlot, S. 2003, MNRAS, 344, 1000

Calzetti, D., Lee, J. C., Sabbi, E., et al. 2015, AJ, 149, 51

Chabrier, G. 2003, PASP, 115, 763

Chandar, R., Whitmore, B. C., Calzetti, D., \& O’Connell, R. 2014, ApJ, 787, 17 
Chandar, R., Fall, S. M., \& Whitmore, B. C. 2010a, ApJ, 711, 1263

Chandar, R., Whitmore, B. C., \& Fall, S. M. 2010b, ApJ, 713, 1343

Chandar, R., Whitmore, B. C., Kim, H., et al. 2010c, ApJ, 719, 966

Dopita, M. A., Calzetti, D., Maíz Apellániz, J., et al. 2010, Ap\& SS, 33

Fall, S. M., Krumholz, M. R., \& Matzner, C. D. 2010, ApJ, 710, L142

Fall, S. M., \& Chandar, R. 2012, ApJ, 752, 96

Fitzpatrick, E. L. 1999, PASP, 111, 63

Fouesneau, M., Lancon, A., Chandar, R., \& Whitmore, B. C. 2012, ApJ, 750, 60

Goddard, Q. E., Bastian, N., \& Kennicutt, R. C. 2010, MNRAS, 405, 857

Harris, J., \& Zaritsky, D. 2004, AJ, 127, 1531

Harris, J., \& Zaritsky, D. 2009, AJ, 138, 1243

Hunter, D., Elmegreen, B. G., Dupuy, T. J., \& Mortonson, M. 2003, AJ, 126, 1836

James, P. A., O’Neill, J., \& Shane, N. S. 2008, A\& A, 486, 131

Kennicutt, R. C. 1998, ARA\& A, 36, 189

Kennicutt, R. C., \& Evans, N. J. 2012, ARA\& A, 50, 531

Kruijssen, J. M. D. 2012, MNRAS, 426, 3008

Lee, J. C., Gil de Paz, A., Tremonti, C., et al. 2009, ApJ, 706, 599

Leitherer, C., Schaerer, D., Goldader, J. D., et al. 1999, ApJS, 123, 3

Lada, C. J., \& Lada, E. A. 2003, ARA\&A, 41, 57

Larsen, S. S. 2002, AJ, 124, 1393

McKee, C. F., \& Ostriker, E. C. 2007, ARA\&A, 45, 565

Raiter, A., Schaerer, D., \& Fosbury, R. A. E. 2010, A\& A, 523, 64

Rangelov, B., Prestwich, A. H., \& Chandar, R. 2011, ApJ, 741, 86

Salpeter, E. 1955, ApJ, 121, 161 
Schweizer, F., Burns, C. R., Madore, B. F., et al. 2008, AJ, 136, 1482

Silva-Villa, E., \& Larsen, S. S. 2010, A\&A, 516, 10

Silva-Villa, E., Adamo, A., \& Bastian, N. 2013, MNRAS, 436, 69

Silva-Villa, E., Adamo, A., Bastian, N., Fouesneau, M., \& Zackrisson, E. 2014, MNRAS, 440, 116

Smith, L. J., Norris, R. P. F., \& Crowther, P. A. 2002, MNRAS, 337, 1309

Thim, F., Tammann, G. A., Saha, A., et al. 2003, ApJ, 590, 256

Whitmore, B. C. 2003, "The Formation of Star Clusters," in A Decade of Hubble Space Telescope Science. ed., Mario Livio, Keith Noll, \& Massimo Stiavelli (Cambridge University Press, Cambridge, UK)

Whitmore, B. C., Chandar, R., \& Kin, H., et al. 2011, ApJ, 729, 78

Zhang, Q., Fall, S. M., \& Whitmore, B. C. 2001, ApJ, 561, 727 


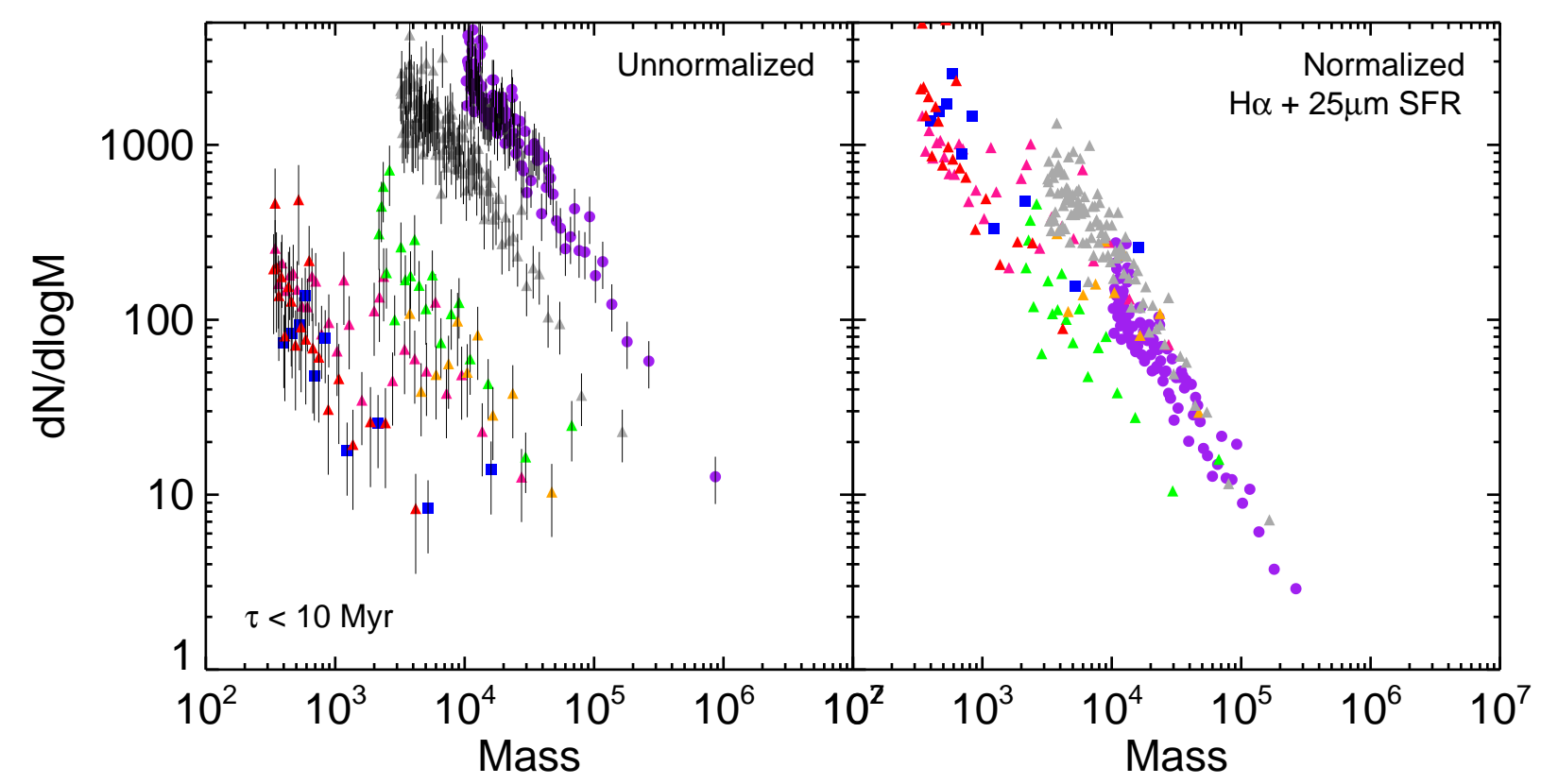

Fig. 1. - Mass functions of star clusters younger than $10 \mathrm{Myr}$ in 7 star-forming galaxies. The symbols are as follows: LMC: pink; SMC: blue; NGC 4214: red; NGC 4449: orange; M83: green; M51: gray; and Antennae: purple. The selection of the clusters is described in the text. The left panel shows the unnormalized mass functions. The right panel shows the mass functions normalized by the current star formation rate (SFR). The fractional coverage of each galaxy is also accounted for. 


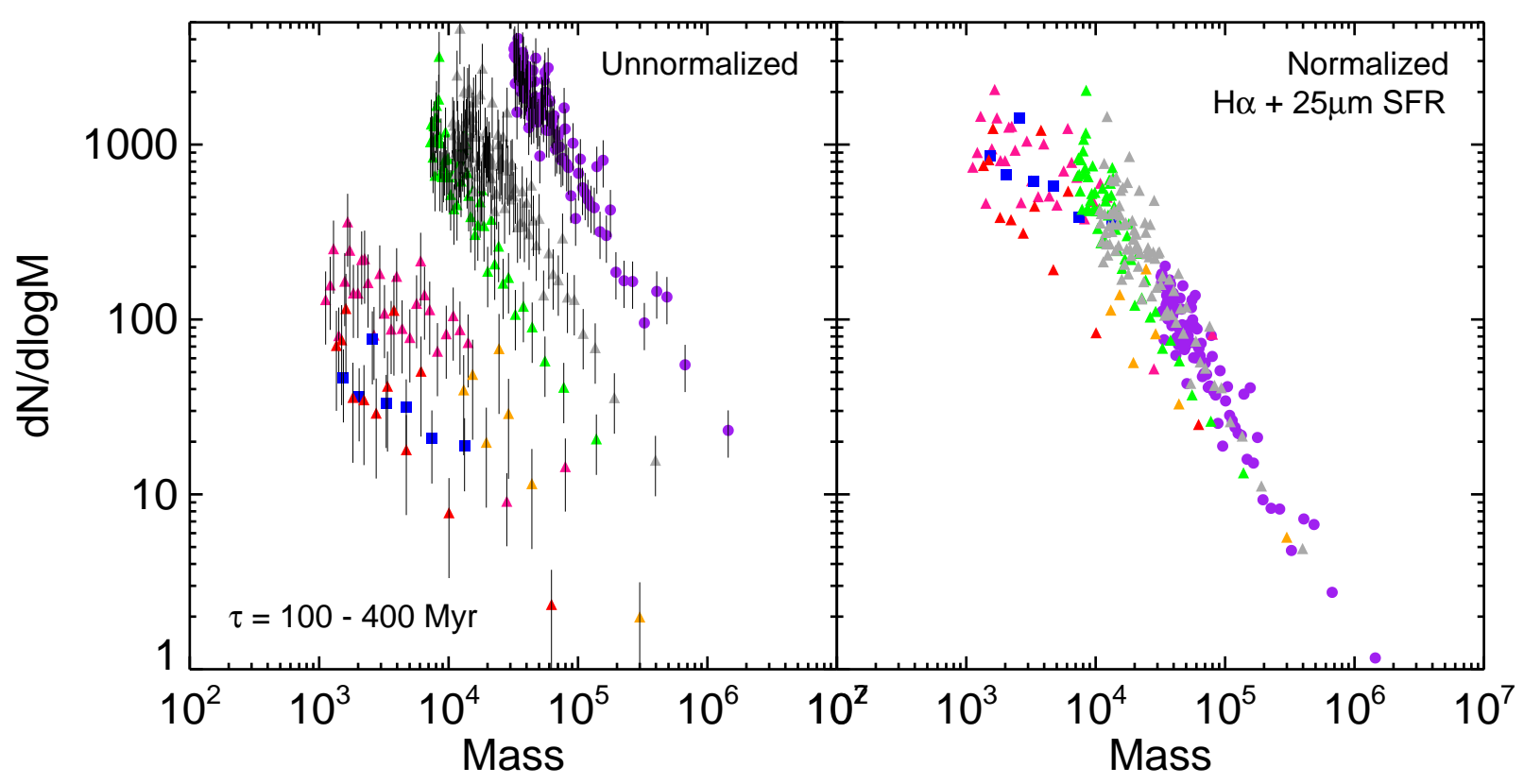

Fig. 2.- Same as Figure 1, but now for clusters in the age range 100-400 Myr.

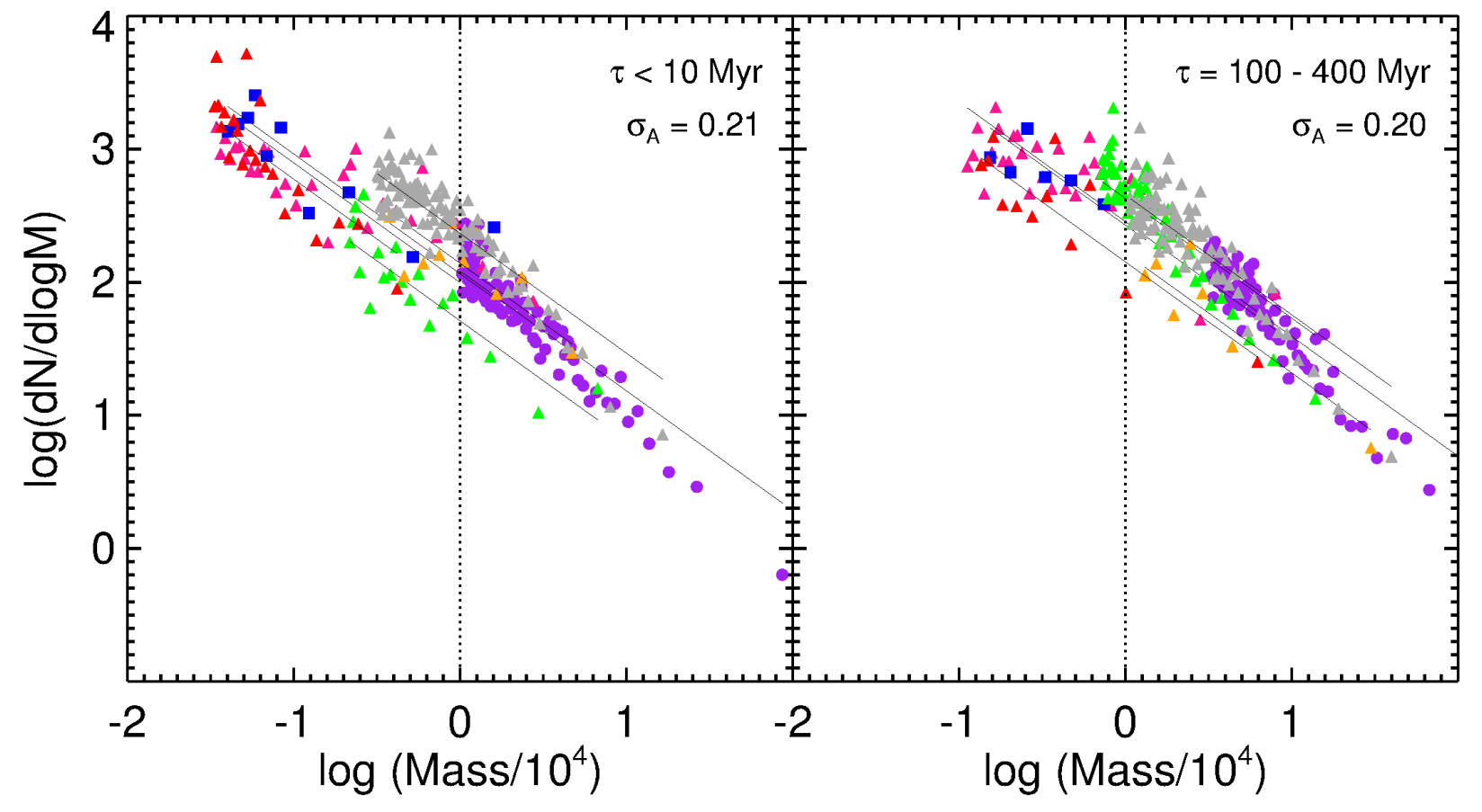

Fig. 3.- Illustration of our method for determining the scatter in the CMF/SFR relation for young clusters $(\tau<10 \mathrm{Myr}$, left panel) and intermediate-age clusters $(\tau=100-400 \mathrm{Myr}$, right panel). The standard deviations of $\log A$ are given in the upper right of each panel (denoted by $\sigma_{A}$ ). 

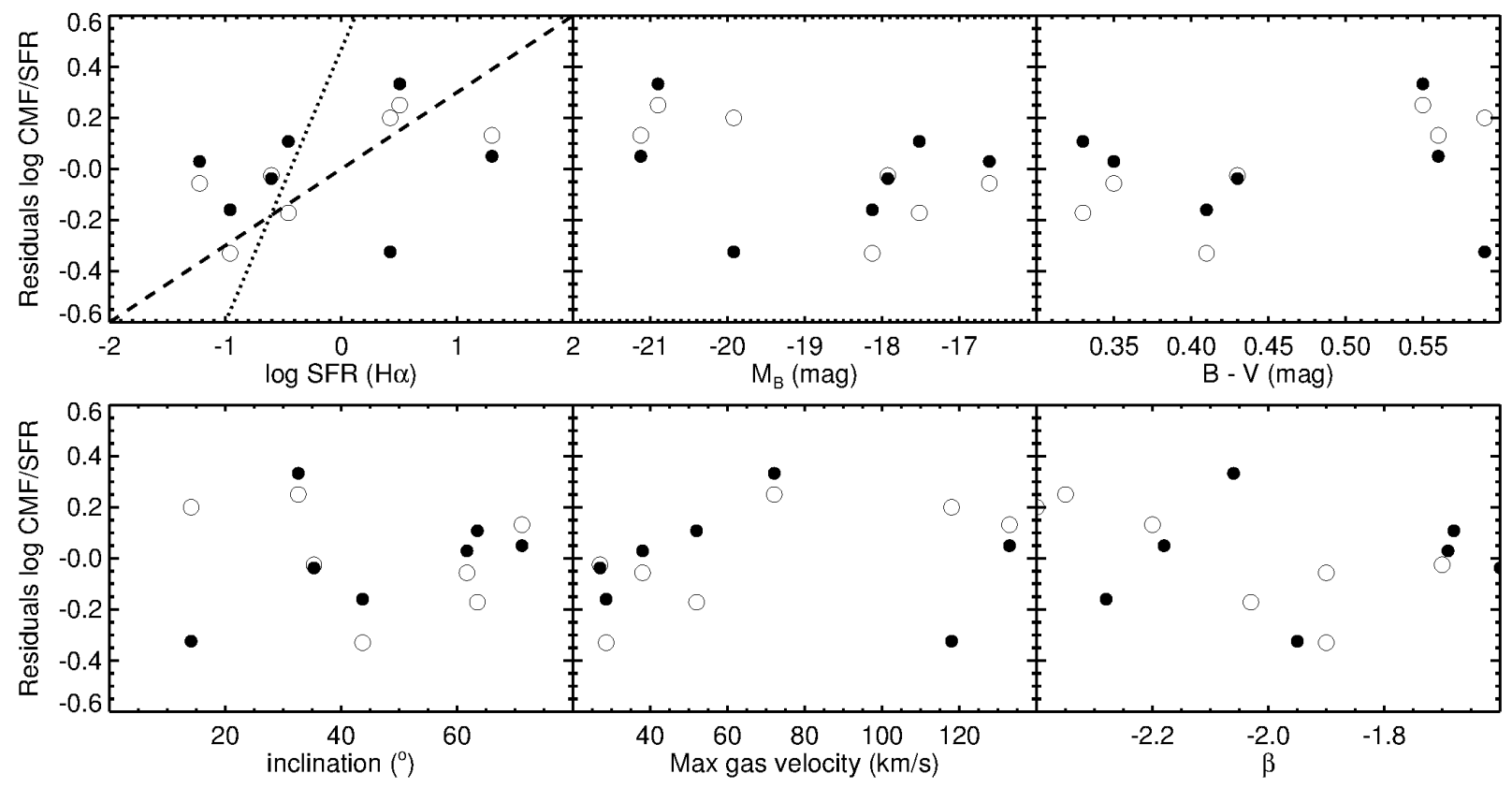

Fig. 4.- Logarithmic residuals in the CMF/SFR relations for young $(\tau \leq 10$ Myr, filled circles) and intermediate-age (100-400 Myr, open circles) clusters plotted against a number of galaxy properties. No statistically significant correlations are found between the residuals and the galaxy properties considered here for the young $\tau<10$ Myr clusters. There are weak trends with galactic SFR, luminosity, and $B-V$ color for the intermediate-age (100$400 \mathrm{Myr}$ ) clusters, but only the one with $B-V$ is statistically significant (at the $95 \%$ level). The top-left panel shows that our results do not agree with the two correlations presented in Kruijssen 2012, which were based on a compilation of observational results (dashed line) and theoretical calculations (dotted line). See text for more details. 
Table 1. Properties of Sample Galaxies

\begin{tabular}{cccccccc}
\hline \hline Galaxy & Galaxy & $\begin{array}{c}\mathrm{H} \alpha+25 \mu \mathrm{m} \\
\mathrm{SFR}{ }^{2} \\
\text { Name }\end{array}$ & Type & $\begin{array}{c}\text { Published } \\
\mathrm{H} \alpha \text { SFR } \\
(\mathrm{Mpc})\end{array}$ & $\begin{array}{c}\text { Published } \\
\beta^{1}\end{array}$ & $\begin{array}{c}\text { Other SFR } \\
\left(M_{\odot} / \mathrm{yr}\right)\end{array}$ & $\begin{array}{c}\text { References } \\
\left(M_{\odot} / \mathrm{yr}\right)\end{array}$ \\
\hline LMC & Irregular & 0.05 & $-1.60 \pm 0.10$ & 0.25 & 0.25 & 0.39 & 1,2 \\
SMC & Irregular & 0.06 & $-1.69 \pm 0.15$ & 0.06 & 0.04 & 0.05 & 3,4 \\
NGC 4214 & Irregular & 3.1 & $-2.28 \pm 0.17$ & 0.11 & 0.16 & 0.22 & 5,5 \\
NGC 4449 & Irregular & 3.8 & $-1.68 \pm 0.21$ & 0.35 & 0.66 & 0.89 & 5,5 \\
M83 & Spiral & 4.5 & $-1.95 \pm 0.12$ & 2.65 & 3.35 & 5.01 & 5,5 \\
M51 & Spiral & 8.2 & $-2.06 \pm 0.05$ & 3.20 & 4.48 & 7.58 & 5,5 \\
Antennae & Merging & 22.2 & $-2.18 \pm 0.04$ & 20.20 & 25.48 & $\ldots$ & 6 \\
\hline
\end{tabular}

${ }^{1}$ Power-law index for the mass function of star clusters, determined from the least-squares fits to $\log (d N / d \log M)=(\beta-1) \log M+$ const

${ }^{2}$ Star formation rates determined in this work. See Section 3.1 for a description

Note. - References: 1. James et al. 2008; 2. Harris \& Zaritsky 2009; 3. Bolatto et al. 2011; 4. Harris \& Zaritsky 2004; 5. Lee et al. 2009; 6. Zhang et al. 2001 
Table 2. Uncertainties in SFR Estimates

\begin{tabular}{cc}
\hline \hline $\begin{array}{c}\text { Source } \\
\text { of Uncertainty }\end{array}$ & $\begin{array}{c}\text { Uncertainty } \\
\log \text { SFR }\end{array}$ \\
\hline Cluster Mass Function & $0.1(\tau<10 \mathrm{Myr})$ \\
Flux Measurements & $0.04(\tau=100-400 \mathrm{Myr})$ \\
Extinction Correction & $\approx 0.04-0.10$ \\
Fractional Coverage & $\approx 0.1$ \\
Lyc Escape Fraction & unknown \\
Metallicity & systematic trend \\
& $\approx 0.4$ at $1 / 10 Z_{\odot}$ \\
\hline
\end{tabular}

Table 3. Spearman Correlation Coefficients for Residuals Shown in Figure 4

\begin{tabular}{crccc}
\hline \hline Galaxy & \multicolumn{3}{c}{ Age Range $\tau<10$ Myr } & \multicolumn{2}{c}{ Age Range $\begin{array}{c}\text { T } \\
\text { Property }\end{array}$} & $\mathrm{r}_{S}$ & $\mathrm{p}\left(>\mathrm{r}_{S}\right)$ & $\mathrm{r}_{S}$ & $\mathrm{p}\left(>\mathrm{r}_{S}\right)$ \\
\hline $\log \mathrm{SFR}\left(M_{\odot} / \mathrm{yr}\right)$ & 0.39 & 0.39 & 0.71 & 0.07 \\
$\mathrm{M}_{B}(\mathrm{mag})$ & -0.11 & 0.82 & -0.66 & 0.10 \\
$B-V(\mathrm{mag})$ & -0.29 & 0.53 & 0.78 & 0.04 \\
inclination $(\mathrm{deg})$ & 0.39 & 0.38 & -0.50 & 0.25 \\
Max gas velocity $(\mathrm{km} / \mathrm{s})$ & 0.25 & 0.59 & 0.61 & 0.15 \\
CMF index $\beta$ & 0.07 & 0.88 & -0.04 & 0.94 \\
\hline
\end{tabular}

Note. - The nonparametric Spearman correlation coefficient, $r_{S}$ and the corresponding probabilities $\mathrm{p}\left(>r_{S}\right)$ are compiled. A perfect correlation would have a value of \pm 1 , and the sign indicates the direction of the association between the listed galaxy properties and the residuals in $\log A$. Values for $\mathrm{p}\left(>r_{S}\right)$ should be less than 0.05 to have greater than $95 \%$ confidence that there is a real correlation. The only correlation that satisfies this condition is the one with $B-V$ for intermediate-age clusters. 